\title{
Experimental recovery of intrinsic fluorescence and fluorophore concentration in the presence of hemoglobin: spectral effect of scattering and absorption on fluorescence
}

Vinh Nguyen Du Le Michael S. Patterson Thomas J. Farrell Joseph E. Hayward Qiyin Fang 


\title{
Experimental recovery of intrinsic fluorescence and fluorophore concentration in the presence of hemoglobin: spectral effect of scattering and absorption on fluorescence
}

\author{
Vinh Nguyen Du Le, ${ }^{a}$ Michael S. Patterson, ${ }^{a, b}$ Thomas J. Farrell, ${ }^{a, b}$ Joseph E. Hayward, ${ }^{a, b}$ and Qiyin Fang ${ }^{c, *}$ \\ ${ }^{a}$ McMaster University, Department of Medical Physics and Applied Radiation Sciences, Hamilton, Ontario L8S 4L8, Canada \\ bJuravinski Cancer Centre, Hamilton, Ontario L8V 5C2, Canada \\ 'McMaster University, Department of Engineering Physics, Hamilton, Ontario L8S 4L8, Canada
}

\begin{abstract}
The ability to recover the intrinsic fluorescence of biological fluorophores is crucial to accurately identify the fluorophores and quantify their concentrations in the media. Although some studies have successfully retrieved the fluorescence spectral shape of known fluorophores, the techniques usually came with heavy computation costs and did not apply for strongly absorptive media, and the intrinsic fluorescence intensity and fluorophore concentration were not recovered. In this communication, an experimental approach was presented to recover intrinsic fluorescence and concentration of fluorescein in the presence of hemoglobin $(\mathrm{Hb})$. The results indicated that the method was efficient in recovering the intrinsic fluorescence peak and fluorophore concentration with an error of $3 \%$ and $10 \%$, respectively. The results also suggested that chromophores with irregular absorption spectra (e.g., $\mathrm{Hb}$ ) have more profound effects on fluorescence spectral shape than chromophores with monotonic absorption and scattering spectra (e.g., black India ink and polystyrene microspheres). ๑ 2015 Society of Photo-Optical Instrumentation Engineers (SPIE) [DOI: 10.1117/1.JBO.20.12.127003]
\end{abstract}

Keywords: intrinsic fluorescence signal; hemoglobin; optical properties; diffuse reflectance; fluorescence quantum yield.

Paper 150515RR received Aug. 1, 2015; accepted for publication Nov. 12, 2015; published online Dec. 22, 2015.

\section{Introduction}

Many biological fluorophores in human mucosal tissues, such as collagen, nicotinamide adenine dinucleotide (NADH), and flavin adenine dinucleotide (FAD), can absorb light at shorter wavelengths (e.g., in the ultraviolet region) and emit fluorescence at longer wavelengths (i.e., in the visible region). ${ }^{1}$ Their fluorescence properties (e.g., intensity and lifetime) and relative concentration can be used to obtain the status of the studied tissues..$^{2-9}$ For example, the fluorescence of NADH and FAD may increase as cells become dysplastic due to the disruption of the extracellular matrix, ${ }^{2,3}$ whereas collagen density or concentration increases during tumor progression. ${ }^{4}$ However, during in vivo measurements, background absorbers and scatterers strongly distort the intrinsic fluorescence and make it difficult to obtain the correct concentration of the fluorophore. The term "intrinsic fluorescence signal" (IFS) was adapted by different research groups to describe the fluorescence without the interference of other absorbers and scatters. ${ }^{10-12}$ Details of the physical definition of IFS can be found in Sec. 2.3 of the manuscript. In soft tissues, typical absorbers are hemoglobin ( $\mathrm{Hb}$, primary absorber), collagen crosslinks, NADH, and FAD (secondary absorbers), whereas scatterers include cell nuclei, organelles, and collagen fibers. ${ }^{5}$ The fluorophore concentration can be extracted only if the effect of absorption and scattering is compensated and the IFS is retrieved.

Many studies have attempted to retrieve the IFS using theoretical modeling, experimental approaches, or a combination of both. For example, Palmer and Ramanujam ${ }^{13}$ developed a Monte-Carlo method incorporated with Mie theory and a nonlinear optimization algorithm to recover the IFS of furan dye within $10 \%$ error. Kanick et al. ${ }^{14}$ extended MC algorithms to investigate the effect of absorption on the measured fluorescence intensity, but the information regarding intrinsic fluorescence and fluorophore concentration was not recovered. MC algorithms were also used to calculate the optical fiber's calibration factor, which was later used to recover the IFS from the measured diffuse reflectance (DR). ${ }^{12}$ Although the MC-based method is flexible for different illumination-collection geometries, it requires intensive computation during forward modeling even if modern computational power is applied. ${ }^{15-21}$ Therefore, it is necessary to develop a simpler approach that can perform fast recovery of fluorescence and be adapted to different systems. In studies by Diamond et al., ${ }^{22}$ the fluorophore concentration was extracted by applying diffusion theory for the measured fluorescence and DR. However, optical properties extraction based on the diffusion approximation may not be accurate for tumorous tissues with high angiogenesis (such as glioblastoma) or for intraoperative surgery where bleeding is unavoidable. In such case, absorption level usually dominates scattering level, and diffuse approximation becomes inapplicable. ${ }^{23}$

Finlay and Foster ${ }^{24}$ used a forward adjoint model of fluorescence to recover the shape of intrinsic fluorescence spectra and $\mathrm{Hb}$ absorption with the prior knowledge of concentration of known absorbers and known fluorophores. The approach, however, was not able to retrieve the absolute fluorescence intensity 
and fluorophore concentration. ${ }^{24}$ In studies by Kim et al., an analytical method was developed to correct the fluorescence line shape and fluorophore concentration based on the assumption that fluorescence is in a linear relationship with DR at emission wavelengths. ${ }^{25}$ However, such an assumption is valid only if an excitation wavelength within 380 to $450 \mathrm{~nm}$ is used so that the migration path of emitted photons is approximately the same as that of reflected photons. ${ }^{25}$ Furthermore, previous phantom studies considered furan 2 as the fluorophores $^{12}$ and red marking dye as the background absorber. ${ }^{16}$ Such materials are not relevant to most tissue investigations, and the fluorescence intensity and fluorophore concentration were not recovered. For example, furan 2 has an emission peak at 400 to $420 \mathrm{~nm}$, whereas many endogenous fluorophores such as NADH, FAD, and elastin have emission peaks at longer wavelengths (450 to $550 \mathrm{~nm}){ }^{1}$ Hoy et al. attempted to recover the intrinsic fluorescence line shape of fluorescein based on the recovered optical properties with the single-fiber fluorescence (SFF) technique. ${ }^{26}$ However, the fluorescence intensity and fluorophore concentration were not recovered for comparison. Moreover, the validation of SFF was performed on phantoms with high concentrations of intralipid while using $365 \mathrm{~nm}$ as the excitation source. ${ }^{26} \mathrm{~A}$ recent report has shown that intralipid fluoresces strongly at this concentration and has a longer fluorescence lifetime (4.5 ns) compared to fluorescein (3.8 to $4 \mathrm{~ns}) .{ }^{1,27}$ Therefore, its fluorescence properties should not be ignored. Although different studies were able to recover the intrinsic fluorescence and concentration of protoporphyrin IX in human skin using excitation light at longer wavelengths from 600 to $800 \mathrm{~nm},{ }^{28-30}$ many fluorophores in human mucosa and brain such as NADH, FAD, and collagen absorb light at shorter wavelengths from 350 to $500 \mathrm{~nm} .^{1}$ Therefore, additional evaluations on phantoms or tissues with $\mathrm{Hb}$ as the dominant absorber are necessary if these approaches ${ }^{28-30}$ are to be applied to image mucosal tissues or brain tissues.

In this paper, we explored the ability of a simpler approach ${ }^{31}$ for fast recovery of fluorescence peaks and fluorophore concentration in highly absorptive media using an excitation wavelength at $355 \mathrm{~nm}$. The approach was validated on tissuesimulating phantoms using fluorescein as fluorophore, polystyrene microspheres as background scatterers, and black India ink and ferrous-stabilized $\mathrm{Hb}$ as background absorbers.

\section{Methods}

\subsection{Tissue-Simulating Phantoms}

Stock solution of fluorescein with a concentration of $10^{-3} \mathrm{M}$ was prepared by dissolving fluorescein powder (46955-100GF, Sigma-Aldrich, Missouri) in concentrated ethanol 95\% (Commercial Alcohols, GreenField Specialty Alcohol Inc., Ontario, Canada). Fluorescein concentrations of $10^{-4}$ and $10^{-5} \mathrm{M}$ were prepared by simply diluting the stock solution in deionized (DI) water. The absorption and emission spectra of fluorescein are extremely sensitive to alcohol residue, and careful sample preparation is necessary to obtain accurate measurements. ${ }^{27}$ To simulate tissue scattering, polystyrene microspheres with diameter of $1 \mu \mathrm{m}$ (07310-15, Polysciences Inc., Pennsylvania) were used. These spheres were preferred because their scattering properties are similar to biological tissues, and their well-controlled size, index of refraction, ${ }^{17,18,32}$ and accurate estimation of scattering properties are calculated using Mie theory. ${ }^{33-35}$ To investigate the effect of scattering on fluorescence, six phantoms (S1 to S6) with six different concentrations of microspheres (from $0.05 \%$ to $0.72 \% \mathrm{w} / \mathrm{v}$ ) were created. These concentrations were obtained by diluting the original concentration of $2.65 \% \mathrm{w} / \mathrm{v}$ in DI water. ${ }^{27}$

To simulate tissue absorption, both black India ink (Higgins Ink, Chartpak Inc., Massachusetts) and Hb (H0267, SigmaAldrich, Missouri) were used. Black India ink is widely used to simulate absorbers in tissue optics because of its similar exponential decrease of absorption with wavelength as that of NADH and FAD, its low cost, spectral stability, and low fluorescence activity. ${ }^{2,5,36-40}$ Applying the same assumption made by previous studies, ${ }^{38,40}$ the current study treated India ink as a pure absorber. Absorption and scattering coefficients of black India ink and microspheres are shown in Sec. 3.2. Ferrous-stabilized $\mathrm{Hb}$ was selected because its absorption spectrum is close to that of human blood, ${ }^{18}$ and due to its high and stable oxygen saturation $(\sim 100 \%))^{33,41}$ Absorption coefficients of $\mathrm{Hb}$ phantoms are shown in Sec. 3.1. A stock solution with $\mathrm{Hb}$ concentration of $30 \mathrm{mg} / \mathrm{ml}$ was prepared by diluting $\mathrm{Hb}$ powder in DI water.

To observe the effect of $\mathrm{Hb}$ absorption on fluorescence, three phantoms with $\mathrm{Hb}$ concentrations of $3.5,10$, and $20 \mathrm{mg} / \mathrm{ml}$ ( $\mathrm{Hb} 3.5, \mathrm{Hb} 10$, and $\mathrm{Hb} 20)$ and microsphere concentration of $0.4 \% \mathrm{w} / \mathrm{v}$ were produced. To investigate the effects of secondary absorbers on fluorescence, five phantoms (I1 to I5) with various India ink concentrations $(0.05 \%$ to $0.6 \%)$ and microsphere concentration of $0.72 \% \mathrm{w} / \mathrm{v}$ were created. In all phantoms, optical properties were controlled and calculated by applying BeerLambert's law to the absorbance of pure solute absorbers (fluorescein, India ink, or $\mathrm{Hb}$ ) measured with a spectrophotometer (Ultraspec 3000, Pharmacia Biotech Inc., New Jersey) for absorption coefficient $\left(\mu_{\mathrm{a}}\right)$ and Mie theory for reduced scattering coefficient $\left(\mu_{\mathrm{s}}^{\prime}\right)$. Scattering anisotropy $(g)$ of polystyrene microspheres can be found in a previous study. ${ }^{27}$ The volume and depth of each phantom were $6 \mathrm{ml}$ and $5.5 \mathrm{~cm}$, respectively. Each phantom was contained in a test tube with diameter of about $12 \mathrm{~mm}$.

\subsection{Instrumentation}

DR signal from the phantoms was generated using a broadband light source (Dolan-Jenner MI-150, Edmund Optics, New Jersey) for illumination, whereas steady state fluorescence (SSF) signal was generated using a solid-state laser (PNV001525-140, Teem Photonics, Meylan, France) at $355 \mathrm{~nm}$ with 300-ps full width at half maximum for excitation. Measurements of both DR and SSF signals were performed with the same customized optical probe. ${ }^{42}$ Figure 1 shows the geometry of the optical probe, including a DR/SSF source fiber; different DR/ SSF detection fibers bundled into three groups at three source-detector distances (SDD) of $0.23,0.59$, and $1.67 \mathrm{~mm}$; and a center fiber for time-resolved fluorescence (TRF) measurement. Three spectrometers (UV-NIR-200, StellarNet Incorporation, Tampa, Florida) were used to record the reflectance signal and SSF signal and were controlled by a moderately equipped computer (IBM Core 2 Duo L7500, 2 GB RAM). The position of the fiber probe was unchanged during both measurements. This enabled the correction of fluorescence using the DR measured at the same location.

Later, the fluorescence lifetime of fluorescein in each phantom was recorded using center optical fiber with a core diameter of $400 \mu \mathrm{m}$ and numerical aperture of 0.12 (Fig. 1) and a calibrated acousto-optic tunable filter-based time-resolved 


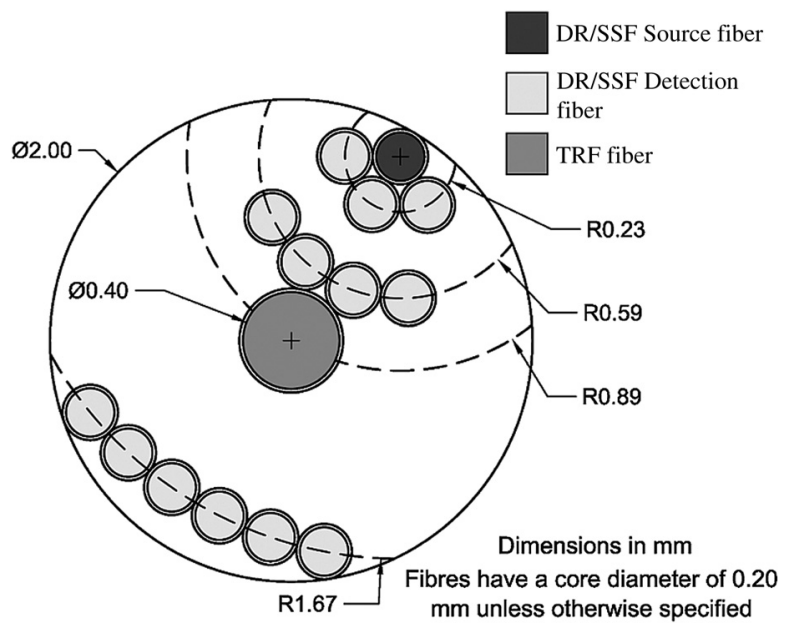

Fig. 1 Schematics of the fiber probe geometry. Diffuse reflectance/ steady-state fluorescence (DR/SSF) detection fibers are bundled together into three groups, each at the indicated distance $(0.23$, 0.89 , and $1.67 \mathrm{~mm}$ ) from the DRS/SSF source fiber (measured from the center of each fiber). In addition, a central fiber was used for time-resolved fluorescence (TRF) spectroscopy measurements. Diameter of DR/SSF fibers is $200 \mu \mathrm{m}$ and diameter of the TRF fiber is $400 \mu \mathrm{m}$.

spectrometer. ${ }^{43}$ Details of the time-resolved system can be found elsewhere. ${ }^{43,44}$ In all measurements, the fiber-optic probe was held perpendicular to the phantom surface and slightly touched the phantoms. The time-resolved measurements merely served as a secondary verification of the stability and consistency in fluorescence properties of fluorescein in all phantoms (the primary verification was spectrometer measurement of absorbance spectra) and had no role in retrieving fluorescein concentration. In the current study, experimental measurements with India ink and microspheres were performed on different days with different batches and concentrations of fluorescein. Therefore, consistency in our time-resolved measurements would also ensure that the use of additional absorbers and scatterers did not alter the chemical structure of fluorescein and that the same alcohol concentration was present in all the phantoms.

\subsection{Retrieving of Intrinsic Fluorescence}

When the excitation light with intensity $I_{x}$ was used to illuminate the sample, fluorescence was emitted with a spectral distribution $f_{x m}\left(\lambda_{m}\right)$. However, due to absorption and scattering in the tissue sample, only a fraction of $f_{x m}$, defined as $F_{x m}\left(\lambda_{m}\right)$, was collected. The subscripts $x$ and $m$ indicate the dependence of the quantity on excitation and fluorescence emission wavelengths $\left(\lambda_{x}\right.$ and $\left.\lambda_{m}\right)$, respectively. Hence, $F_{x m}$ and $f_{x m}$ represent the measured fluorescence and intrinsic fluorescence at emission wavelength $m$ due to excitation wavelength $x$, respectively. Müller et al. and Zhang et al. defined $f_{x m}$ as the total fluorescence intensity of a thin slab of thickness $l(\mathrm{~cm})$ of a material that does not absorb or scatter photons while containing the same concentration of fluorophores. ${ }^{12,16,31}$ In a closed form, $f_{x m}$ can be written in terms of Eq. (1), where $\mu_{a f x}$ is the absorption coefficient $\left(\mathrm{cm}^{-1}\right)$ due only to the slab's fluorophore at excitation wavelength $x$, and $\phi_{x m}$ (dimensionless) is the fluorescence quantum yield. ${ }^{12,16,31}$

$f_{x m}=\frac{\lambda_{x}}{\lambda_{m}} I_{x} l \mu_{a f x} \phi_{x m}$.
In Eq. (1), ratio $\lambda_{x} / \lambda_{m}$ corrects for the difference in photon energy at excitation and emission wavelength, whereas the product $l \mu_{a f x}(\ll 1)$ represents the probability of a photon at excitation wavelength $\lambda_{x}$ to be absorbed by the fluorophore, and $\phi_{x m}$ represents the probability of a photon at emission wavelength $\lambda_{m}$ to be generated after the absorption event at $\lambda_{x}$. $\mu_{\text {afx }}$ can be defined as $c \varepsilon \ln (10)$, where $c$ is fluorophore molar concentration (M) and $\varepsilon$ extinction coefficient $\left(\mathrm{cm}^{-1} \mathrm{M}^{-1}\right)$ at $\lambda_{x}$. Because $f_{x m}$ directly relates to $c$, Eq. (1) can be rewritten in the form of Eq. (2) with a consideration of a mixture of $i$ fluorophores:

$f_{x m}=\frac{\lambda_{x}}{\lambda_{m}} I_{x} l \ln (10) \sum_{i} \varepsilon_{i} c_{i} \phi_{x m}^{i}$.

By using the escape probability distribution function $(\rho)$, the phase-function dependence of the scattering was separated from the scattering and absorption coefficients, and the diffuse reflectance $(R)$ and the measured fluorescence $\left(F_{x m}\right)$ can be modeled using Eqs. (3) and (4). ${ }^{12,31}$

$R=\sum_{n=1}^{\infty} \rho_{n} w_{n}$

$F_{x m}=\sum_{n=1}^{\infty} \sum_{i=0}^{n-1} \rho_{n i} w_{n i}$

where $w_{n}=a^{n}=\left[\mu_{\mathrm{s}} /\left(\mu_{\mathrm{a}}+\mu_{\mathrm{s}}\right)\right]^{n}, w_{n i}=a_{x}^{i+1}\left(f_{x m} / \mu_{s x}\right) a_{m}^{n-i-1}$, and $\rho_{n}=R_{0}\left[e^{S(1-g)}-1\right] e^{-n S(1-g)}$.

In Eqs. (3) and (4), $n$ is the number of scattering events (dimensionless), $S$ is a constant calibration factor for each individual fiber (dimensionless), $w$ is the photon weight (dimensionless), $a$ is the albedo (dimensionless), $\mu_{\mathrm{a}}$ is the absorption coefficient $\left(\mathrm{cm}^{-1}\right), \mu_{\mathrm{s}}$ is the scattering coefficient $\left(\mathrm{cm}^{-1}\right), g$ is the anisotropy parameter (dimensionless), and $R_{0}$ is the diffuse reflectance from a standard sample containing only scatterers (dimensionless). By combining the expressions of $R$ and $F_{x m}$ into Eq. (1), a relationship between $f_{x m}$ and $F_{x m}$ can be written as Eq. (5).

$$
f_{x m}=\frac{\mu_{s x} * l * F_{x m}}{\left\{\frac{R_{0 x} R_{0 m}}{\left[e^{S\left(1-g_{x}\right)}-1\right]\left[e^{S\left(1-g_{m}\right)}-1\right]}\right\}^{1 / 2} \frac{R_{t x}}{R_{0 x}}\left\{\frac{R_{t m}}{R_{0 m}}+\left[e^{S\left(1-g_{m}\right)}-1\right]\right\}} .
$$

In Eq. (5), $R_{t}$ is the diffuse reflectance from the targeted samples. In the studied phantoms, $\mu_{\mathrm{s}}$ and $g$ were obtained by applying Mie theory for specific microsphere diameter and concentration. ${ }^{27}$ To calculate the diffuse reflectance $R$, the measured reflectance intensity of the sample $I_{\mathrm{r} \text { sample }}$ was normalized to the reflectance intensity of a standard spectralon sample with $99.9 \%$ reflectivity $I_{\text {r,std }}$ (Labsphere, Inc., New Hampshire) after subtracting the background signal intensity $I_{\mathrm{r}, \mathrm{bg}}$. This method is described via Eq. (6).

$R=\frac{I_{\mathrm{r}, \mathrm{sample}}-I_{\mathrm{r}, \mathrm{bg}}}{I_{\mathrm{r}, \mathrm{std}}-I_{\mathrm{r}, \mathrm{bg}}}$.

The retrieved signal $f_{x m}$ in Eq. (5) can be used to recover the concentration of fluorophore for a known value of $\phi_{x m}$. In the current study, fluorescence quantum yield of fluorescein in basic 
ethanol was assumed to be 0.97 at emission peak. ${ }^{45,46}$ In experimental measurement, due to the strong intensity of the laser, a neutral density filter with optical density of 2 and a short exposure time $(\sim 1 \mathrm{~ms})$ was applied when measuring $I_{x}$ to avoid saturation at spectrometers. Therefore, to enable accurate estimation of $c$ from Eq. (2), a correction factor $k$ was applied so that $k=10^{-2}(1 / 1130)$, where $1 / 1130$ is the ratio of exposure time used for $I_{x}$ and for $F_{x m}$ (or $f_{x m}$ ). The concentration $c$ of fluorescein in the phantom can be experimentally estimated in Eq. (7):

$$
c=k \frac{f_{x m} \lambda_{m}}{I_{x} \lambda_{x} l \varepsilon \phi_{x m} \ln (10)} .
$$

To obtain the calibration factors $l$ and $S$ in Eq. (5), the least squares fitting routine fminsearch () in MATLAB ${ }^{\circledR}$ was used to fit the measurement of $f_{x m}$ (from the reference nonscattering phantom), $F_{x m}$, and $R_{x m}$ of calibration phantoms into Eq. (5). This optimization method is based on the NelderMead simplex algorithm and has been widely used for spectral analysis in spectral imaging. ${ }^{47-49}$ In the fitting routine, the ideal intrinsic fluorescence $f_{x m}$ was measured directly on a phantom consisting solely of fluorescein $10^{-4} \mathrm{M}$ (in DI water), whereas $F_{x m}$ and $R_{x m}$ were measured with phantoms consisting of fluorescein $10^{-4} \mathrm{M}$ and various India ink and microsphere concentrations. To simplify the fitting routine, $l$ was set at $1 \mathrm{~cm}$. Fitting routines for known spectra of $f_{x m}, F_{x m}$, and $R_{x m}$ yielded values of 54.6, 45.5, and 48.2 for fibers with an SDD of 0.23, 0.59, and $1.67 \mathrm{~mm}$, respectively. These $S$ values were later used to retrieve $f_{x m}$ in $\mathrm{Hb}$ phantoms.

Similar to previous methods, ${ }^{12,31}$ the current method utilized the ideas that enabled the retrieving of intrinsic fluorescence from the measured DR. The main difference lies in the calibration approaches. The current method incorporated system characteristics such as light source intensity and position of the fiber probe into the calibration. Therefore, the current method enabled recovery of absolute fluorescence intensity via Eq. (5) and of fluorophore concentration via Eq. (7), whereas the previous methods could recover only the relative fluorescence intensity and spectral shape.

\section{Results}

\subsection{Effect of Hemoglobin Absorption on Fluorescence}

Figure 2 shows $\mu_{\mathrm{a}}$ of $\mathrm{Hb}$ and fluorescein, as well as $\mu_{\mathrm{s}}^{\prime}$ of polystyrene micropsheres used in the Hb-based phantoms (Hb3.5, $\mathrm{Hb} 10$, and $\mathrm{Hb} 20)$. In these phantoms, the concentration of fluorescein and microspheres remained the same, whereas $\mathrm{Hb}$ concentration was varied. The inset of Fig. 2(b) shows that the measured $\mu_{\mathrm{a}}$ was in excellent agreement with reference $\mu_{\mathrm{a}}$ values for fluorescein $10^{-4} \mathrm{M}$ in alcohol. ${ }^{45,50}$ The reference $\mu_{\mathrm{a}}$ was calculated by multiplying fluorescein concentration to that of its tabulated molar extinction coefficients. ${ }^{50}$ As indicated elsewhere, an $\mathrm{Hb}$ concentration of $3.5 \mathrm{mg} / \mathrm{ml}$ best simulates background absorption in mucosal tissue. ${ }^{18}$ In addition, a microsphere concentration of $0.4 \% \mathrm{w} / \mathrm{v}(\sim 0.0073$ spheres per cubic micrometer) was used to simulate background scattering.

Figure 3(a) shows the measured SSF $\left(F_{x m}\right)$ from three phantoms using SDD of $0.59 \mathrm{~mm}$. Due to the similarity in the measured fluoresence spectra at different fibers, only the signal at one fiber is shown [Fig. 3(a)]. In general, increasing absorption (from 3.5 to $20 \mathrm{mg} / \mathrm{ml}$ ) decreases the measured fluorescence intensity due to the decrease in the number of emitted photons escaping to the tissue surface. To compare the spectral shapes, the fluorescence spectrum of fluorescein in DI water (instrinsic) and in $\mathrm{Hb}$ and microspheres (distorted) is shown in the same graph [Fig. 3(b)]. As shown in Fig. 3(b), the spectral shape of the measured fluorescence was highly distorted in the wavelength regions where $\mathrm{Hb}$ strongly absorbs photons $(540 \mathrm{~nm}$ and $580 \mathrm{~nm}$ ). Figure 3(c) shows examples of the absorption dependence of the measured fluorescence at the fluorescein emission peak of $520 \mathrm{~nm}$.

Despite the distortion in the measured SSF, it was observed that average lifetime in the phantoms remains unchanged, indicating that lifetime was not affected by background absorption (Fig. 4). A biexponential deconvolution method was used to obtain the lifetime information of the measured fluorescence signals. ${ }^{43}$ Figure 4 (a) shows that fluorescence lifetime is consistent at the emission wavelength for a phantom with $\mathrm{Hb}$ concentration of $3.5 \mathrm{mg} / \mathrm{ml}$. In addition, Fig. 4(b) shows that the fluorescence lifetime of fluorescein in $\mathrm{Hb}$ phantoms agreed with that of the intrinsic signal within one standard deviation. In Fig. 4(b), the fluorescence lifetime was averaged over six repeated measurements, and standard deviations were calculated and shown as error bars.

Figure 5(a) shows the corresponding DR of three Hb-based phantoms using SDD of $0.59 \mathrm{~mm}$. In general, the drops of reflectance at the 540 and $580 \mathrm{~nm}$ regions were due to $\mathrm{Hb}$ absorption in these regions [Fig. 2(a)], whereas the drop at 470 to $520 \mathrm{~nm}$ was mainly due to fluorescein absorption [Fig. 2(b)]. Figures 5(b) and 5(c) compare the measured fluorescence $\left(F_{x m}\right)$ to that of recovered and intrinsic fluoresence for a phantom with $\mathrm{Hb}$ concentration of $3.5 \mathrm{mg} / \mathrm{ml}$ using SDD of $0.59 \mathrm{~mm}$. The recovered fluoresence $\left(f_{x m 1}\right)$ is the fluorescence signal calculated using Eq. (5). The least-square fitting routine fminsearch () was also applied to fit $f_{x m 1}$ to the shape of the intrinsic spectrum (ideal) and obtain the fitted spectrum $f_{x m 2}$ of $f_{x m 1}$. The square errors between $f_{x m 1}$ and $f_{x m 2}$ were used to optimize the results of the fitting. Figure 5(d) compares the percentage difference among $F_{x m}, f_{x m 1}$, and $f_{x m 2}$ with respect to the ideal intrinsic fluorescence for phantom $\mathrm{Hb} 3.5$ (Hb concentration of $3.5 \mathrm{mg} / \mathrm{ml}$ ).

On average over 480 to $620 \mathrm{~nm}$, the percentage error was $\sim 81 \%, 24 \%$, and $8 \%$ for $F_{x m}, f_{x m 1}$, and $f_{x m 2}$, respectively.
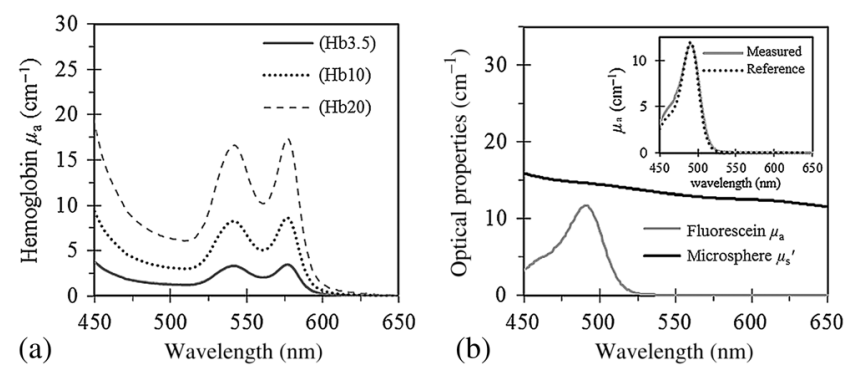

Fig. 2 (a) Absorption coefficients $\mu_{\mathrm{a}}$ of hemoglobin $(\mathrm{Hb})$ phantoms (Hb3.5: $3.5 \mathrm{mg} / \mathrm{ml}, \mathrm{Hb} 10: 10 \mathrm{mg} / \mathrm{ml}$, and $\mathrm{Hb} 20: 20 \mathrm{mg} / \mathrm{ml}$ ) as a function of wavelength and (b) reduced scattering coefficients $\mu_{\mathrm{s}}^{\prime}$ of polystyrene microspheres and $\mu_{\mathrm{a}}$ of fluorescein as a function of wavelength. In these phantoms, the concentration of microsphere and fluorescein was kept constant at $0.4 \% \mathrm{w} / \mathrm{v}$ and $10^{-4} \mathrm{M}$, respectively. The inset in (b) compares $\mu_{\mathrm{a}}$ of fluorescein $10^{-4} \mathrm{M}$ measured in the current study (measured) to that extracted from the literature (reference)..$^{50}$ 

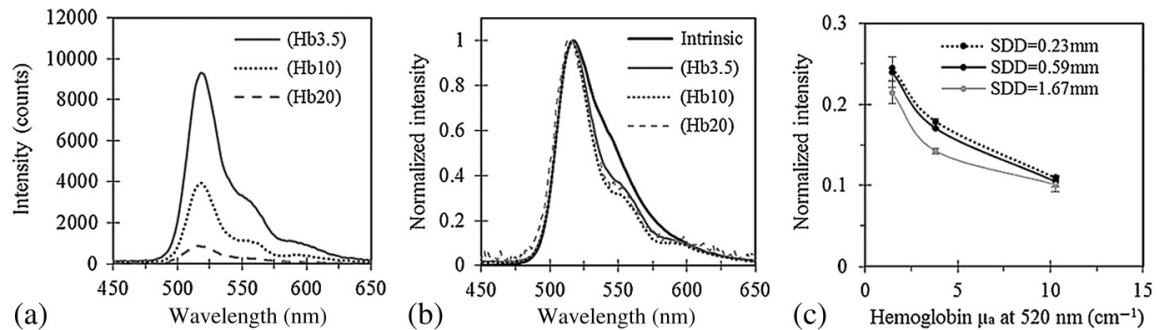

Fig. 3 (a) Measured fluorescence $\left(F_{x m}\right)$ of phantoms ( $\mathrm{Hb3} .5, \mathrm{Hb} 10$, and $\left.\mathrm{Hb} 20\right)$, (b) normalized emission spectra, and (c) normalized intensity as a function of $\mu_{\mathrm{a}}$ at the emission peak $520 \mathrm{~nm}$ for all three collection distances. In (c), the intensity at the emission peak of phantoms was normalized to that of the sample consisting solely of fluorescein $10^{-4} \mathrm{M}$ in deionized water (DI) water (intrinsic). In all cases, the concentration of fluorescein was $10^{-4} \mathrm{M}$; microsphere was $0.4 \% \mathrm{w} / \mathrm{v}$, respectively; and SDD of $0.59 \mathrm{~mm}$ was used.
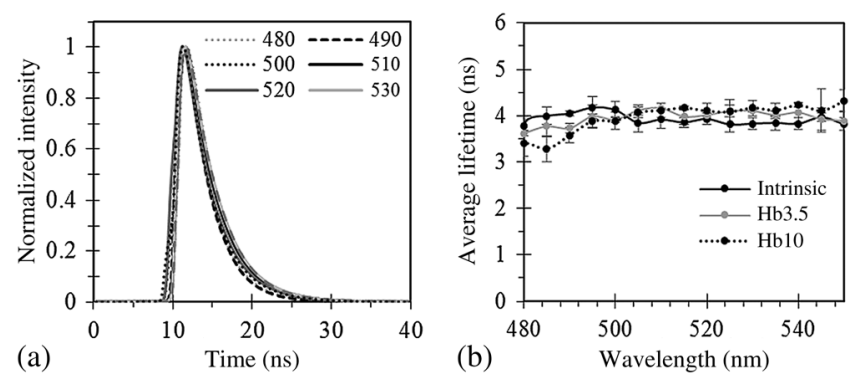

Fig. 4 (a) Fluorescence lifetime for phantom (Hb3.5) at selected emission wavelengths and (b) average fluorescence lifetime of the phantoms ( $\mathrm{Hb} 3.5$ and $\mathrm{Hb} 10)$. The intrinsic signal was collected using fluorescein $10^{-4} \mathrm{M}$ in diluted ethanol without additional scatterer or absorber.
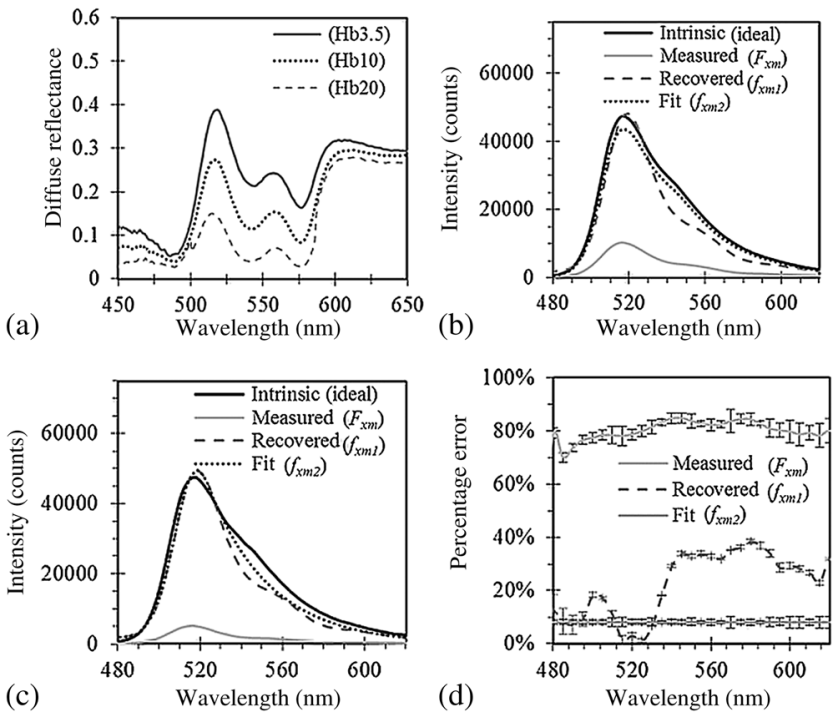

Fig. 5 (a) DR of the phantoms $\mathrm{Hb} 3.5, \mathrm{Hb} 10$, and $\mathrm{Hb} 20$; (b) measured fluorescence $\left(F_{x m}\right)$, the retrieved fluorescence $\left(f_{x m 1}\right)$, the fitted fluorescence $\left(f_{x m 2}\right)$, and the ideal intrinsic fluorescence for phantoms with $\mathrm{Hb}$ concentrations of $3.5 \mathrm{mg} / \mathrm{ml}$ and (c) of $10 \mathrm{mg} / \mathrm{ml}$; (d) Absolute percentage difference in signal intensity with respect to the ideal intrinsic signal for $\mathrm{Hb} 3.5$ phantom. In all cases, fluorescein concentration of $10^{-4} \mathrm{M}$, microsphere concentration of $0.4 \% \mathrm{w} / \mathrm{v}$, and SSD of $0.59 \mathrm{~mm}$ were used. In (b) and (c), $F_{x m}$ is fluorescence under the influence of $\mathrm{Hb}$ absorption and microsphere scattering, whereas $f_{x m 1}$ is the recovered fluorescence using Eq. (3), $f_{x m 2}$ is the fitted spectrum of $f_{x m 1}$, and the intrinsic signal is the signal measured in ideal conditions in which the phantom consists solely of fluorescein $10^{-4} \mathrm{M}$ in DI water.
These numbers were $91 \%, 26 \%, 9 \%$ and $99 \%, 30 \%, 13 \%$ for phantoms $\mathrm{Hb} 10$ and $\mathrm{Hb} 20$, respectively (Table 1). On average over 480 to $620 \mathrm{~nm}$, the accuracy of the signal was improved by $70 \%$ without fitting and by $90 \%$ with fitting. Considering only peak emission at $522 \mathrm{~nm}$, the percentage errors of $F_{x m}, f_{x m 1}$, and $f_{x m 2}$ are $79 \%, 3 \%$, and $8 \%$, respectively. The recovered fluorescence at $522 \mathrm{~nm}$ was used to calculate the concentration of fluorescein using Eq. (7). With $x$ of $355 \mathrm{~nm}$ (excitation peak) and $m$ of $522 \mathrm{~nm}$ (emission peak), the values of $I_{355}$ and $f_{355,522}$ are 61225 counts and 45093 counts, respectively. The extinction coefficient $\varepsilon_{355}$ of fluorescein was assumed to be $0.04 \mathrm{~cm}^{-1} \mathrm{M}^{-1},{ }^{50}$ and $\phi_{355,522}$ was assumed to be $0.97 .{ }^{45}$ Therefore, the $c$ value of $1.07 * 10^{-4} \mathrm{M}$ was estimated using Eq. (7). Table 2 summarizes the extracted concentration of fluorescein in three $\mathrm{Hb}$ phantoms. On average, the extracted concentration was within $10 \%$ of the controlled value of $10^{-4} \mathrm{M}$.

\subsection{Effect of India Ink Absorption on Fluorescence}

To further investigate the absorption effect on fluorescence, black India ink with increasing concentration from phantoms I1 to I5 was used to simulate background absorption and a microsphere concentration of $0.72 \%$ was used to simulated background scattering. Figure 6 summarizes the optical properties of the phantoms used in this section. The goal of this subsection is to investigate the effects of a monotonic decreasing absorption on fluorescence intensity and spectral shape, and to compare it to the $\mathrm{Hb}$ cases.

Figure 7 (a) summarizes the measured fluorescence $\left(F_{x m}\right)$ from phantoms I1 to I5, whereas Fig. 7(b) compares the spectral shape of $F_{x m}$ and $f_{x m}$, and Fig. 7(c) summarizes the corresponding $R$. These data were collected at $\mathrm{SDD}=0.59 \mathrm{~mm}$. A similar trend to that of Fig. 3(a) was observed in Fig. 7(a):

Table 1 Average percentage difference (AVG) of $F_{x m}, f_{x m 1}, f_{x m 2}$ with respect to the ideal intrinsic fluorescence over 490 to $620 \mathrm{~nm}$. Analysis for all three phantoms ( $\mathrm{Hb} 3.5, \mathrm{Hb} 10$, and $\mathrm{Hb} 20)$ is shown.

\section{Percentage error (\%) for each phantom}

\begin{tabular}{lccc}
\cline { 2 - 4 } Quantity & $\mathrm{Hb} 3.5$ & $\mathrm{Hb} 10$ & $\mathrm{Hb} 20$ \\
\hline$F_{x m}$ & $80.8 \pm 2.7$ & $91.3 \pm 1.8$ & $98.7 \pm 0.4$ \\
$f_{x m 1}$ & $24.1 \pm 12.4$ & $26.3 \pm 11.4$ & $29.6 \pm 11.1$ \\
$f_{x m 2}$ & $8.1 \pm 1.5$ & $8.5 \pm 0.8$ & $12.8 \pm 1.1$ \\
\hline
\end{tabular}


Table 2 The recovered fluorescein concentration (c) in three hemoglobin $(\mathrm{Hb})$ phantoms from Eq. (5). The controlled concentration of fluorescein in these phantoms was $10^{-4} \mathrm{M}$.

\begin{tabular}{lccc} 
& \multicolumn{3}{c}{ Phantom } \\
\cline { 2 - 4 } Quantity & $\mathrm{Hb3.5}$ & $\mathrm{Hb} 10$ & $\mathrm{Hb} 20$ \\
\hline$f_{355,522}$ (counts) & $45,093 \pm 786$ & $49,958 \pm 232$ & $44,174 \pm 317$ \\
$F_{355,522}$ (counts) & $9472 \pm 165$ & $4527 \pm 21$ & $557 \pm 4$ \\
$c(\mathbf{M})$ & $\mathbf{1 . 0 7 E - 0 4}$ & $\mathbf{1 . 1 9 E}-04$ & $\mathbf{1 . 0 5 E}-04$ \\
& $4 . \mathrm{E}-06$ & $2 . \mathrm{E}-06$ & $2 . \mathrm{E}-06$ \\
\hline
\end{tabular}
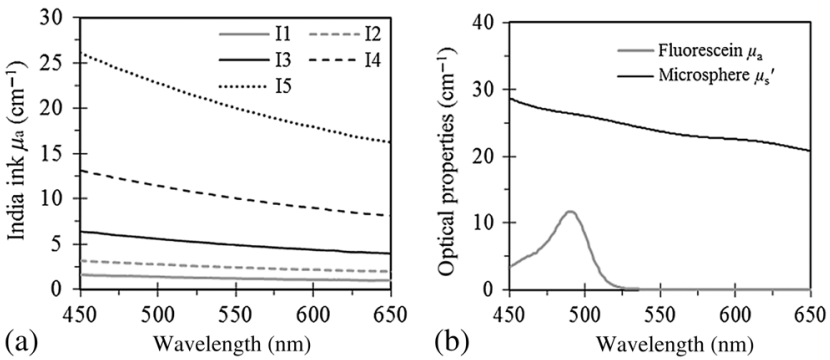

Fig. 6 (a) Absorption coefficients $\mu_{\mathrm{a}}$ of the India ink in phantoms I1 to 15 as a function of wavelength and (b) reduced scattering coefficients $\mu_{\mathrm{s}}^{\prime}$ of polystyrene microsphere and $\mu_{\mathrm{a}}$ of fluorescein as a function of wavelength. In these phantoms, the concentration of India ink increased from phantom 11 to 15 , and the concentration of microsphere and the fluorescein was kept constant at $0.72 \% \mathrm{w} / \mathrm{v}$ and $10^{-4} \mathrm{M}$, respectively.
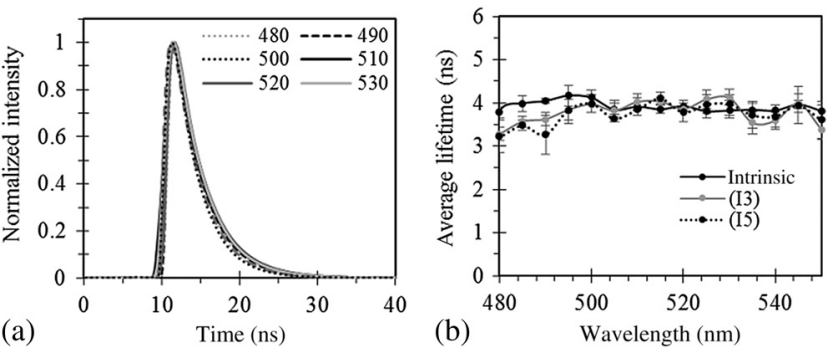

Fig. 8 (a) Fluorescence lifetime for phantom (I3) at selected emission wavelengths and (b) average lifetime of the phantoms (I3 and I5) at emission wavelength. The intrinsic signal was collected using fluorescein $10^{-4} \mathrm{M}$ in diluted ethanol without additional scatterer or absorber.

SSF decreases as absorption increases. However, there is a significant difference between Figs. 3(b) and 7(b): the spectral shape of the fluorescence for phantoms with ink as background absorber was not distorted and remained similar to that of the intrinsic signal (measured with only fluorescein in DI water). This can be explained by referring to either the India ink absorption spectra [Fig. 6(a)] or the phantom DR spectra [Fig. 7(c)]. Similar to the $\mathrm{Hb}$ phantom cases, increasing absorption (from I1 to I5) decreases the reflectance signal collected [Fig. 7(c)]. However, in contrast to the $\mathrm{Hb}$ phantoms, there are no peaks in the India ink absorption spectra [Fig. 6(a)] or DR spectra [Fig. 7(c)]. Note that the drop of DR at $500 \mathrm{~nm}$ in Fig. 7(c) was due to the strong absorption of fluoresecein at this region. Again, Eq. (7) can be applied to extract fluorescein concentration. An average error of 5\% was obtained for the recovered fluorescein concentration (Table 3). Furthermore, the fluorescence lifetime of India ink phantoms remained the same as that of the intrinsic signal, as expected (Fig. 8). Table 4 provides a brief summary of the average fluoresence lifetime of fluorescein in $\mathrm{Hb}$ and India ink phantoms over the emission region of
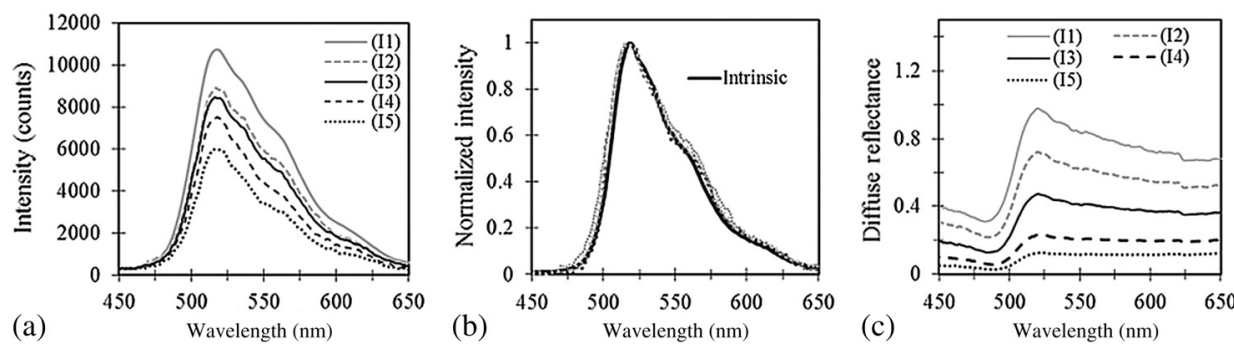

Fig. 7 (a) Measured fluorescence emission spectra of the phantoms (I1 to I5), (b) normalized fluorescence emission spectra, and (c) the corresponding DR spectra. These data were collected with the fiber at $\mathrm{SDD}=0.59 \mathrm{~mm}$. In all phantoms, fluorescein concentration was $10^{-4} \mathrm{M}$.

Table 3 The recovered fluorescein concentration in ink-microsphere phantoms. The controlled concentration of fluorescein in these phantoms was $10^{-4} \mathrm{M}$.

Phantom

\begin{tabular}{lccccc}
\cline { 2 - 5 } Quantity & I1 & I2 & I3 & 14 & I5 \\
\hline$f_{355,522}$ (counts) & $43,652 \pm 741$ & $46,230 \pm 420$ & $46,589 \pm 744$ & $42,442 \pm 837$ & $41,342 \pm 823$ \\
$F_{355,522}$ (counts) & $10,479 \pm 149$ & $8778 \pm 58$ & $8282 \pm 112$ & $7281 \pm 115$ & $5918 \pm 93$ \\
$c(\mathrm{M})$ & $\mathbf{1 . 0 4 E - 0 4}$ & $\mathbf{1 . 1 0 E}-\mathbf{0 4}$ & $\mathbf{1 . 1 1 E}-\mathbf{0 4}$ & $\mathbf{1 . 0 1 E - 0 4}$ & $\mathbf{9 . 8 4 E - 0 5}$ \\
& $3 . \mathrm{E}-06$ & $2 . \mathrm{E}-06$ & $3 . \mathrm{E}-06$ & $3 . \mathrm{E}-06$ & $3 . \mathrm{E}-06$ \\
\hline
\end{tabular}


Table 4 Overall average lifetime of fluorescein in different phantoms (in region 480 to $550 \mathrm{~nm}$ ). Concentration of fluorescein in these phantoms was $10^{-4} \mathrm{M}$. Concentration of microspheres was $0.4 \% \mathrm{w} / \mathrm{v}$ and $0.72 \% \mathrm{w} / \mathrm{v}$ in $\mathrm{Hb}$ and India ink phantoms, respectively.

Phantom

\begin{tabular}{lccccccc}
\cline { 2 - 7 } Quantity & Intrinsic & Hb3.5 & Hb10 & Hb20 & (I3) & (I4) & (I5) \\
\hline$\tau$ (ns) & $3.92 \pm 0.12$ & $3.96 \pm 0.16$ & $3.97 \pm 0.31$ & $3.95 \pm 0.32$ & $3.78 \pm 0.26$ & $3.84 \pm 0.25$ & $3.74 \pm 0.26$ \\
\hline
\end{tabular}

480 to $550 \mathrm{~nm}$. Overall, the fluoresence lifetime remained constant and agreed with previously reported values for fluorescein in diluted alcohol. ${ }^{1}$

\subsection{Effect of Microsphere Scattering on Fluorescence}

Figure 9 summarizes the optical properties of phantoms S1 to S6. In these phantoms, microsphere concentration increases
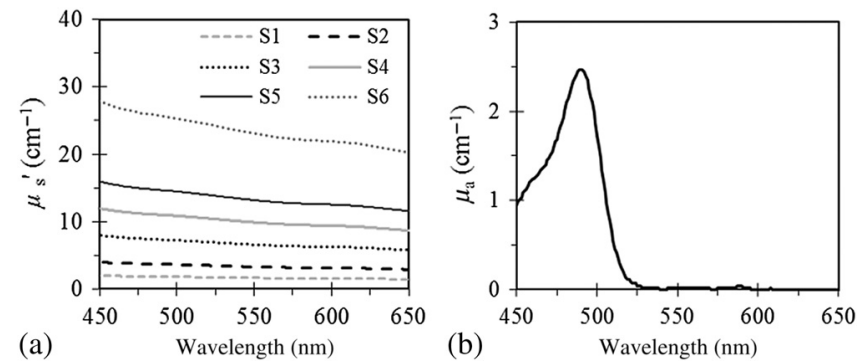

Fig. 9 (a) Reduced scattering coefficients $\mu_{\mathrm{s}}^{\prime}$ and (b) absorption coefficients $\mu_{\mathrm{a}}$ of phantoms $\mathrm{S} 1$ to $\mathrm{S} 6$. In these phantoms, the concentration of microspheres increased from phantoms $\mathrm{S} 1$ to $\mathrm{S6}$, whereas the concentration of fluorescein was kept at $10^{-5} \mathrm{M}$. No other absorbers were added. from S1 $(0.05 \% \mathrm{w} / \mathrm{v})$ to S6 $(0.72 \% \mathrm{w} / \mathrm{v})$ [Fig. 9(a)], whereas the concentration of fluorescein is kept constant at $10^{-5} \mathrm{M}$. No other absorbers were added. Figure 9(b) shows the $\mu_{\mathrm{a}}$ spectrum of fluorescein $10^{-5} \mathrm{M}$ solution. The goal of this subsection is to inspect the effects of a monotonically decreasing reduced scattering on fluorescence intensity and spectral shape.

The measured SSF of the phantoms is shown in Fig. 10 for all collection distances: $0.23 \mathrm{~mm}$ [Fig. 10(a)], $0.59 \mathrm{~mm}$ [Fig. 10(b)], and $1.69 \mathrm{~mm}$ [Fig. 10(c)]. In general, adding microspheres decreased the fluorescence intensity at first due to the effect of scattering (intrinsic versus S1). The fluorescence intensity eventually increased if more microspheres were added from S1 to S6. Figure 11(a) further illustrates this trend by plotting the normalized intensity at the emission peak as a function of $\mu_{\mathrm{s}}^{\prime}$ (at $520 \mathrm{~nm})$. This phenomenon was consistent with what was reported previously. ${ }^{12}$ To compare the intensity trend between SDDs, the fluorescence collected at each SDD was normalized to the intrinsic fluorescence at the same SDD [Fig. 11(a)]. The current results indicated that increasing SDD from 0.23 to $0.59 \mathrm{~mm}$ might increase the fluorescence signal up to $40 \%$ in highly scattering media, although further increasing of SDD showed the reverse [Fig. 11(a)].

The corresponding DR of phantoms for SDD $=0.59 \mathrm{~mm}$ is also shown in Fig. 11(b), and the normalized measured
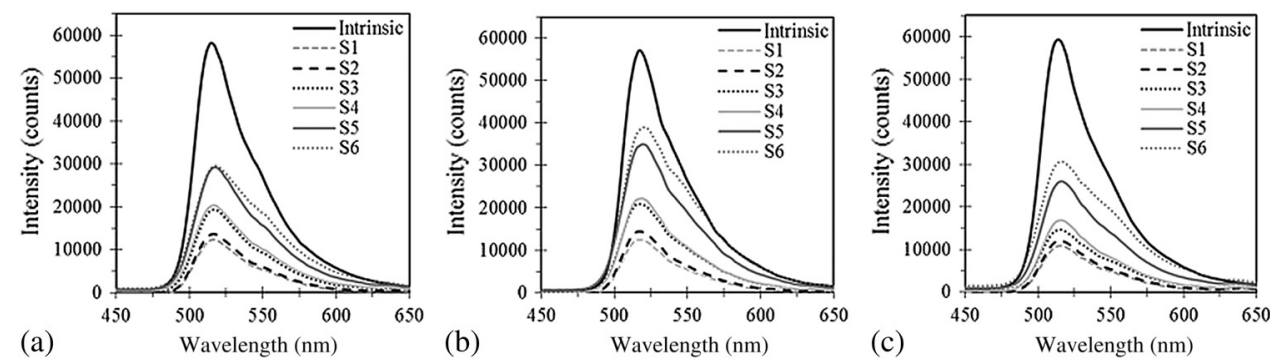

Fig. 10 Measured fluorescence emission spectra of the phantoms using (a) SDD $=0.23 \mathrm{~mm}$, (b) $\mathrm{SDD}=0.59 \mathrm{~mm}$, and (c) $\mathrm{SDD}=1.67 \mathrm{~mm}$. Microsphere concentration increased from sample $\mathrm{S} 1$ to sample S6. Fluorescein concentration was kept constant at $10^{-5} \mathrm{M}$.
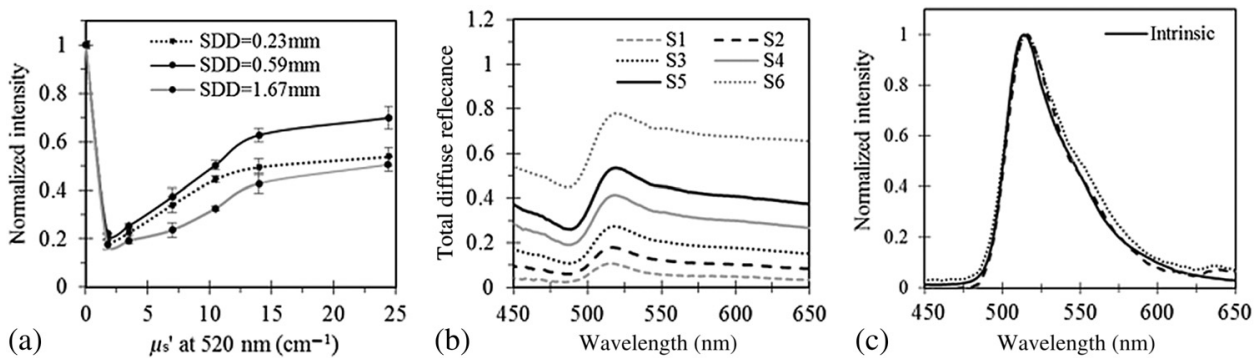

Fig. 11 (a) Normalized fluorescence intensity as a function of $\mu_{\mathrm{s}}^{\prime}$ at emission peak of $520 \mathrm{~nm}$ for all three SDDs, (b) corresponding total DR spectra at SDD $=0.59 \mathrm{~mm}$, and (c) normalized fluorescence intensity. Fluorescein concentration was kept constant at $10^{-5} \mathrm{M}$. 
Table 5 The extracted fluorescein concentration (c) in microsphere phantoms from Eq. (5). The controlled concentration of fluorescein in these phantoms was $10^{-5} \mathrm{M}$.

Phantom

\begin{tabular}{lcccccc}
\cline { 2 - 6 } Quantity & S1 & S2 & S3 & S4 & S5 & S6 \\
\hline$f_{355,522}$ (counts) & $40,550 \pm 1247$ & $51,902 \pm 872$ & $43,682 \pm 945$ & $54,050 \pm 462$ & $49,208 \pm 1145$ & $51,367 \pm 916$ \\
$F_{355,522}$ (counts) & $10,036 \pm 211$ & $10,998 \pm 178$ & $13,493 \pm 258$ & $15,688 \pm 160$ & $24,617 \pm 377$ & $29,294 \pm 236$ \\
$c$ (M) & $9.05 \mathrm{E}-06$ & $\mathbf{1 . 1 6 E}-05$ & $9.75 \mathrm{E}-06$ & $\mathbf{1 . 2 1 E}-05$ & $\mathbf{1 . 1 0 E}-05$ & $\mathbf{1 . 1 5 E}-05$ \\
& $6 . \mathrm{E}-07$ & $4 . \mathrm{E}-07$ & $4 . \mathrm{E}-07$ & $2 . \mathrm{E}-07$ & $4 . \mathrm{E}-07$ & $3 . \mathrm{E}-07$ \\
\hline
\end{tabular}
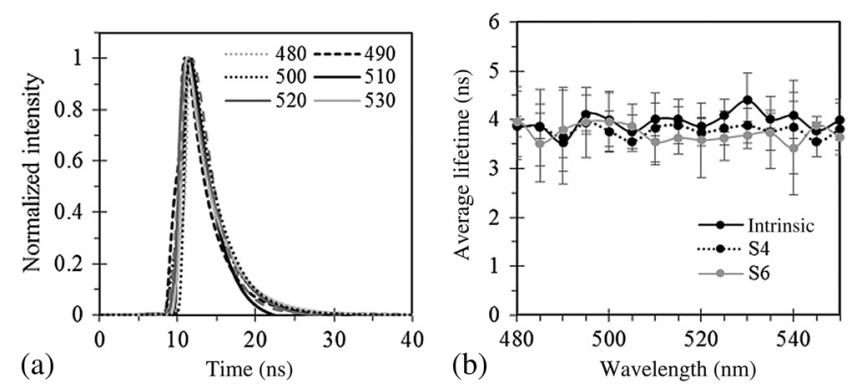

Fig. 12 (a) Fluorescence lifetime for phantom S6 at selected emission wavelengths and (b) average lifetime of the phantoms S4 and S6 at emission wavelength. The intrinsic signal was collected using fluorescein $10^{-5} \mathrm{M}$ in diluted ethanol without additional scatterer or absorber.

fluorescence and intrinsic signal is plotted in Fig. 11(c). In general, increasing scattering increases the probability of detection, thus increasing the reflectance intensity to be collected by the detection fiber [Fig. 11(b)]. This is a reversed trend of increasing absorption in India ink cases [Fig. 7(c)]. Similar to the India ink cases, the spectral shape of the fluorescence in microsphere phantoms remains unchanged [Fig. 11(c)]. The India ink absorption coefficient and microsphere-reduced scattering coefficient are related to wavelength in the form of $\mu=a \lambda^{-b}$, where a and $\mathrm{b}$ are positive fitting coefficients, $\lambda$ is the wavelength, and $\mu$ represents absorption coefficients in the India ink case and reduced scattering coefficients in the microsphere case. ${ }^{34,35}$ Therefore, the results shown in Figs. 7(b) and 11(c) might indicate that exponential decrease of scattering or absorption with increasing wavelengths does not have effects on the fluorescence spectral shape. Equation (7) was applied to retrieve the concentration of fluorescein in the phantoms $\mathrm{S} 1$ to $\mathrm{S} 6$, considering $\mathrm{SDD}=$ $0.59 \mathrm{~mm}$, exposure time ratio of $1 / 11700$, and $I_{x}$ value of 63056 counts. As shown in Table 5, an average percentage error of $8 \%$ was observed in the recovered fluorescein concentration. In addition, the fluorescence lifetime remained within the standard deviation (Fig. 12). Table 6 provides an overall summary of the average lifetime in regions from 480 to $550 \mathrm{~nm}$ for phantoms S1 to S6.

\section{Discussion and Conclusions}

The current study has shown that $\mathrm{Hb}$ absorption strongly distorted the fluorescence spectra of fluorescein at emission wavelength regions near 540 and $580 \mathrm{~nm}$. Most endogenous fluorophores in human tissues emit in this wavelength region upon irradiation with ultraviolet light. ${ }^{1}$ Using the proposed method and the constructed phantoms with optical property ranges reported for mucosal tissues and brain tissues $\left(\mu_{\mathrm{a}}\right.$ of 1.2 to $20 \mathrm{~cm}^{-1}$ and $\mu_{\mathrm{s}}^{\prime}$ of 1.7 to $24.5 \mathrm{~cm}^{-1}$ at $\left.540 \mathrm{~nm}\right),{ }^{2,5,18,33-35,51,52}$ fluorescein concentration was recovered with an average error of $10 \%$ (Fig. 5, Table 1), considering only the emission peak at $522 \mathrm{~nm}$. On average over 450 to $650 \mathrm{~nm}$, the intrinsic fluorescence intensity of fluorescein was recovered with an error of $27 \%$ (versus $90 \%$ with raw signal). Although spectral fitting reduced error in recovering fluorescence by $66 \%$ on average over 450 to $650 \mathrm{~nm}$, the percentage error of fitted data was higher than that of nonfitted data at the emission peak $522 \mathrm{~nm}$ (3\% versus $8 \%$ ). Although absorption affects fluorescence and DR differently due to the difference in light path shown in Eqs. (3) and (4), this difference was accounted for in Eq. (5). In addition, the absorption distortion on the $f_{x m 2}$ line shape was accounted for by the ratio of $R_{\mathrm{tm}} / R_{0 \mathrm{~m}}$ in Eq. (1). ${ }^{12}$ The proposed method was also efficient in retrieving fluorescein's concentration in India ink and microsphere phantoms with an average percentage error of $5 \%$ and $8 \%$, respectively (Tables 3 and 4). The current approach used the same concept as previous studies to the extent to which the measured DR was used to compensate the effect of $\mathrm{Hb}$ absorption on fluorescence signal. ${ }^{12,31}$ What made our approach unique were the abilities to recover absolute fluorescence intensity and to estimate fluorophore concentration via the experimental calibration of individual fibers without the expense of complicated computation. Our ultimate goal is to recover fluorophore concentration using DR and fluorescence measurements and optical properties of the targeted volume. In this manuscript, we demonstrated that the proposed method is feasible to recover fluorophore concentration if

Table 6 Overall average lifetime in region 480 to $550 \mathrm{~nm}$ of fluorescein for phantoms S1 to S6.

Phantom

\begin{tabular}{lccccccr}
\cline { 2 - 6 } Quantity & Intrinsic & S1 & S2 & S3 & S4 & S5 & S6 \\
\hline$\tau$ (ns) & $3.96 \pm 0.20$ & $3.85 \pm 0.18$ & $3.69 \pm 0.20$ & $3.73 \pm 0.19$ & $3.81 \pm 0.12$ & $3.71 \pm 0.21$ & $3.72 \pm 0.18$ \\
\hline
\end{tabular}


optical properties are known. In the next step, an inverse solution is being developed to recover optical properties and to demonstrate that this approach is able to perform real-time tissue measurement. This addition will make the current approach more practical in studying tissue samples. Although a single fluorophore was used in our study, this should not limit the application of the proposed method in media with multiple fluorophores. As demonstrated in the current study, the recovered intensity at the emission peak is crucial to extract fluorophore concentration. In biological tissues, each fluorophore (NADH, FAD, and collagen) has a known emission peak, which can be recovered using Eq. (1), allowing extraction of its concentration via Eq. (7). Although Müller and Hendriks ${ }^{16}$ were unable to recover fluorophore concentration, their studies demonstrated the ability of the proposed method to recover multiple fluorescence peaks via Eq. (1).

The current results also show that scattering and absorption materials whose attenuation coefficients decay exponentially with wavelengths (such as black India ink and polystyrene microspheres) have a minor effect on the spectral shape of the fluorescence [Figs. 7(b) and 11(c)]. These observations were made when studying the broadband emission signal of fluorescein in 500 to $625 \mathrm{~nm}$. This finding might indicate that absorption and scattering from NADH, FAD, and collagen fibers do not have significant effects on the fluorescence spectral shape because their attenuation coefficients decay exponentially with wavelength, similar to those of India ink or microspheres. ${ }^{2,5,34}$ Therefore, $\mathrm{Hb}$ is the only absorber of concern. In addition, a consistency in the average fluorescence lifetime measured with the time-resolved system verified that the chemical structure of fluorescein was not affected by the introduction of India ink, microspheres, or $\mathrm{Hb}$. Furthermore, the current results suggested that increasing SSD from 0.23 to $0.59 \mathrm{~mm}$ has the potential to increase the fluorescence signal in highly scattering media, especially for S4 to S6 [Fig. 11(a)]. Therefore, a small increase in collection distance could increase the probability of detection. However, a larger increase of SDD would decrease the probability of an emitted photon reaching the detection fiber. As a result, fluorescence intensity was high at SDD of $0.59 \mathrm{~mm}$ and low at SDD of $1.67 \mathrm{~mm}$. Additional theoretical analysis is necessary to confirm this result and to optimize the design to maximize the measured fluorescence signal.

\section{Acknowledgments}

The authors would like to thank professor Leyla Soleymani of the Department of Engineering Physics at McMaster University for access to her laboratory during phantom fabrication. This project was supported in part by the Natural Sciences and Engineering Research Council (NSERC) of Canada and Canadian Cancer Society Research Institute (CCSRI). Q. F. holds the Canada Research Chair in Biophotonics at McMaster University.

\section{References}

1. M. Y. Berezin and S. Achilefu, "Fluorescence lifetime measurements and biological imaging," Chem. Rev. 110(5), 2641-2684 (2010).

2. R. Drezek et al., "Autofluorescence microscopy of fresh cervical-tissue sections reveals alterations in tissue biochemistry with dysplasia," Photochem. Photobiol. 73(5), 636-641 (2001).

3. I. Pavlova et al., "Microanatomical and biochemical origins of normal and precancerous cervical autofluorescence using laser-scanning fluorescence confocal microscopy," Photochem. Photobiol. 77(5) 550-555 (2003).
4. P. P. Provenzano et al., "Collagen density promotes mammary tumor initiation and progression," BMC Med. 6(11), 11 (2008).

5. S. K. Chang et al., "Analytical model to describe fluorescence spectra of normal and preneoplastic epithelial tissue: comparison with Monte Carlo simulations and clinical measurements," J. Biomed. Opt. 9(3), 511-522 (2004).

6. D. C. Walker et al., "A study of the morphological parameters of cervical squamous epithelium,” Physiol. Meas. 24(1), 121-135 (2003).

7. M. H. Johnston, "Technology insight: ablative techniques for Barrett's esophagus: current and emerging trends," Nat. Clin. Pract. Gastroenterol. Hepatol. 2(7), 323-330 (2005).

8. P. V. Butte et al., "Intraoperative delineation of primary brain tumors using time-resolved fluorescence spectroscopy," J. Biomed. Opt. 15(2), 027008 (2010).

9. L. Marcu et al., "Detection of rupture-prone atherosclerotic plaques by time-resolved laser induced fluorescence spectroscopy," Atherosclerosis 204(1), 156-164 (2009).

10. J. R. Lakowicz et al., "Intrinsic fluorescence from DNA can be enhanced by metallic particles," Biochem. Biophys. Res. Commun. 286(5), 875-879 (2001).

11. J. Wu, M. S. Feld, and R. P. Rava, "Analytical model for extracting intrinsic fluorescence in turbid media," Appl. Opt. 32(19), 3585-3595 (1993).

12. M. G. Müller et al., "Intrinsic fluorescence spectroscopy in turbid media: disentangling effects of scattering and absorption," Appl. Opt. 40(25), 4633-4646 (2001).

13. G. M. Palmer and N. Ramanujam, "Monte-Carlo-based model for the extraction of intrinsic fluorescence from turbid media," J. Biomed. Opt. 13(2), 024017 (2008).

14. S. C. Kanick et al., "Extraction of intrinsic fluorescence from single fiber fluorescence measurements on a turbid medium," Opt. Lett. 37(5), 948-950 (2012).

15. G. M. Palmer and N. Ramanujam, "Monte Carlo-based inverse model for calculating tissue optical properties. Part I: theory and validation on synthetic phantoms," Appl. Opt. 45(5), 1062-1071 (2006).

16. M. Müller and B. H. Hendriks, "Recovering intrinsic fluorescence by Monte Carlo modeling," J. Biomed. Opt. 18(2) 027009 (2013).

17. Q. Wang et al., "Condensed Monte Carlo modeling of reflectance from biological tissue with a single illumination-detection fiber," IEEE Sel. Top. Quantum Electron. 16(3) 627-634 (2010).

18. V. N. Du Le et al., "Vascular contrast in narrow band and white light imaging," Appl. Opt. 53(18), 4061-4071 (2014).

19. Q. Fang and D. A. Boas, "Monte Carlo simulation of photon migration in 3D turbid media accelerated by graphics processing units," Opt. Express 17(22), 20178-20190 (2009).

20. J. Chen, Q. Fang, and X. Intes, "Mesh-based Monte Carlo method in time-domain widefield fluorescence molecular tomography," J. Biomed. Opt. 17(10) 106009 (2012).

21. Q. Fang, "Mesh-based Monte Carlo method using fast ray-tracing in Plücker coordinates," Biomed. Opt. Express 1(1), 165-175 (2010).

22. K. R. Diamond, T. J. Farrell, and M. S. Patterson, "Measurement of fluorophore concentrations and fluorescence quantum yield in tissuesimulating phantoms using three diffusion models of steady-state spatially resolved fluorescence," Phys. Med. Biol. 48(24), 4135-49 (2003).

23. D. J. Cuccia et al., "Quantitation and mapping of tissue optical properties using modulated imaging," J. Biomed. Opt. 14, 024012 (2009).

24. J. C. Finlay and T. H. Foster, "Recovery of hemoglobin oxygen saturation and intrinsic fluorescence with a forward-adjoint model," App. Opt. 44(10), 1917-1933 (2005).

25. A. Kim et al., "Quantification of in vivo fluorescence decoupled from the effects of tissue optical properties using fiber-optic spectroscopy measurements," J. Biomed. Opt. 15(6), 067006 (2010).

26. C. L. Hoy et al., "Method for rapid multidiameter single-fiber reflectance and fluorescence spectroscopy through a fiber bundle," J. Biomed. Opt. 18(10), 107005 (2013).

27. V. N. Du Le et al., "Measurements of extrinsic fluorescence in intralipid and polystyrene microspheres," Biomed. Opt. Express 5(8), 2726-2735 (2014)

28. T. A. Middelburg et al., "Correction for tissue optical properties enables quantitative skin fluorescence measurements using multi-diameter single fiber reflectance spectroscopy," J. Dermatol. Sci. 79(1), 64-73 (2015). 
29. R. B. Saager et al., "Quantitative fluorescence imaging of protoporphyrin IX through determination of tissue optical properties in the spatial frequency domain," J. Biomed. Opt. 16(12), 126013 (2011).

30. U. Sunar et al., "Quantification of PpIX concentration in basal cell carcinoma and squamous cell carcinoma models using spatial frequency domain imaging," Biomed. Opt. Express 4(4), 531-537 (2013).

31. Q. Zhang et al., "Turbidity-free fluorescence spectroscopy of biological tissue," Opt. Lett. 25(19), 1451-1452 (2000).

32. B. W. Pogue and M. S. Patterson, "Review of tissue simulating phantoms for optical spectroscopy, imaging and dosimetry," J. Biomed. Opt. 11(4), 041102 (2006).

33. N. Rajaram et al., "Design and validation of a clinical instrument for spectral diagnosis of cutaneous malignancy," Appl. Opt. 49(2), 142152 (2010).

34. Q. Wang et al., "Broadband ultraviolet-visible optical property measurement in layered turbid media," Biomed. Opt. Express 3(5), 1226-1240 (2012).

35. N. Rajaram, T. H. Nguyen, and J. W. Tunnell, "Lookup table-based inverse model for determining optical properties of turbid media," J. Biomed. Opt. 13(5), 050501 (2008).

36. T. R. Wagner, W. G. Houf, and F. P. Incropera, "Radiative property measurements for India ink suspensions of varying concentration," Solar Energy 25, 549-554 (1980).

37. H. Xu and M. Patterson, "Determination of the optical properties of tissue-simulating phantoms from interstitial frequency domain measurements of relative fluence and phase difference," Opt. Express 14(14), 6485-6501 (2006).

38. Q. Liu, C. Zhu, and N. Ramanujam, "Experimental validation of Monte Carlo modeling of fluorescence in tissues in the UV-visible spectrum," J. Biomed. Opt. 8(2), 223-236 (2003).

39. S. A. Ermilov et al., "Laser optoacoustic imaging system for detection of breast cancer," J. Biomed. Opt. 14(2), 024007 (2009).

40. I. Barman et al., "Rapid and accurate determination of tissue optical properties using least-squares support vector machines," Biomed. Opt. Express 2(3), 592-599 (2011).

41. B. Yu et al., "Diffuse reflectance spectroscopy of epithelial tissue with a smart fiber-optic probe," Biomed. Opt. Express 5(3), 675-689 (2014).

42. D. Cappon et al., "Fiber-optic probe design and optical property recovery algorithm for optical biopsy of brain tissue," J. Biomed. Opt. 18(10), 107004 (2013).

43. Z. Nie et al., "Hyperspectral fluorescence lifetime imaging for optical biopsy," J. Biomed. Opt. 18(9), 096001 (2013).

44. Y. Yuan et al., "High throughput AOTF-based time-resolved fluorescence spectrometer for optical biopsy," Opt. Lett. 34(7), 1132-1134 (2009).

45. P. G. Seybold, M. Gouterman, and J. Callis, "Calorimetric, photometric and lifetime determinations of fluorescence yields of fluorescein dyes," Photochem. Photobiol. 9(3), 229-242 (1969).
46. D. Magde, R. Wong, and P. G. Seybold, "Fluorescence quantum yields and their relation to lifetimes of rhodamine $6 \mathrm{G}$ and fluorescein in nine solvents: improved absolute standards for quantum yields," Photochem. Photobiol. 75(4), 327-334 (2002).

47. S. L. Jacques, R. Samatham, and N. Choudhury, "Rapid spectral analysis for spectral imaging," Biomed. Opt. Express 1(1) 157-164 (2010).

48. M. S. Twardowski et al., "Modeling the spectral shape of absorption by chromophoric dissolved organic matter," Mar. Chem. 89(1), 69-88 (2004).

49. X. Zhong, X. Wen, and D. Zhu, "Lookup-table-based inverse model for human skin reflectance spectroscopy: two-layered Monte Carlo simulations and experiments," Opt. Express 22(2), 1852-1864 (2014).

50. J. M. Dixon, M. Taniguchi, and J. S. Lindsey, "PhotochemCAD. A computer-aided design and research tool in photochemistry and photobiology," Photochem. Photobiol. 81(1), 212-213 (2005).

51. R. Nachabé et al., "Estimation of biological chromophores using diffuse optical spectroscopy: benefit of extending the UV-VIS wavelength range to include 1000 to $1600 \mathrm{~nm}$," Biomed. Opt. Express 1(5), 1432-1442 (2010).

52. S. L. Jacques, "Optical properties of biological tissues: a review," Phys. Med. Biol. 58(11), R37 (2013).

Vinh Nguyen Du Le is a PhD student in the medical physics program at McMaster University (Hamilton, ON). He received a BSc degree (2009) and a MSc degree (2010) in biomedical engineering from the Catholic University of America (Washington, DC). He was a research fellow at the U. S. Food and Drug Administration from 2010 to 2012.

Michael S. Patterson is the director of medical physics at Juravinski Cancer Centre (Hamilton, ON). He received a BSc degree (1973) from Queen's University, a MSc degree (1976) from McMaster University, and a PhD degree (1984) from the University of Toronto.

Thomas J. Farrell is a medical physicist at the Juravinski Cancer Centre and the chair of the medical physics department at McMaster University. He received his PhD degree (1990) from McMaster University.

Joseph E. Hayward is a medical physicist at the Juravinski Cancer Centre and an associate professor of the medical physics department at McMaster University. He received his $\mathrm{PhD}$ degree (1993) in lasers and electro-optics in engineering physics from McMaster University.

Qiyin Fang is an associate professor of Engineering Physics and the chair of biophotonics at McMaster University. He received his BSc degree (1995) in physics from Nankai University, his MSc degree (1998) in applied physics, and his PhD degree (2002) in biomedical physics from East Carolina University. 